\title{
Stereotactic Re-irradiation for Recurrent or Second Primary Head-and-Neck Cancer
}

\author{
IIlhami ÜNAL, ${ }^{1}$ Mustafa CENGiZ² \\ 'Department of Radiation Oncology, Ankara Ataturk Training and Research Hospital, Ankara-Turkey \\ ${ }^{2}$ Department of Radiation Oncology, Hacettepe University School of Medicine, Ankara-Turkey
}

\begin{abstract}
SUMMARY
Local recurrence after definitive radiotherapy for advanced head-and-neck cancer is observed in $30 \%-$ $60 \%$ of the patients. Surgical resection is possible in only about $25 \%$ of the cases. Re-irradiation for local recurrence is most of the times the only local treatment option. However, it is highly morbid with a poor success rate. Stereotactic radiotherapy is a highly conformal radiotherapy technique, usually with hypofractionation. Most of the authors use 5-6 fractions by 6-8 Gy. Median OS rates vary between 12 and 24.5 months. Concomitant use of cetuximab may also have some beneficial effects. Recent multicentric RPA analysis from North America suggested the classification of patients into prognostic groups and advised selection of treatment protocols according to the RPA class of the patients. The authors also compared IMRT with SBRT for re-irradiation. They could not show any significant difference between the treatment techniques. Carotid artery blowout syndrome is one of the lethal toxicities of re-irradiation. Limiting radiation dose to the carotid artery is important for the prevention of such toxicities. However, there is currently no consensus pertaining to carotid artery doses in the literature.
\end{abstract}

Keywords: Head and neck cancer; reirradiation; SBRT.

Copyright $\odot$ 2018, Turkish Society for Radiation Oncology

\section{Introduction}

\section{Re-irradiation, SBRT, Head-and-Neck Cancer}

Treatment of head-and-neck cancer (HNC) is performed by surgery, radiotherapy, and chemotherapy modalities alone or together. Nonetheless, $30 \%-60 \%$ of patients develop local recurrence or secondary cancer in the irradiated field.[1]

Re-irradiation is a potentially curative treatment option for some patients with unresectable disease. However, increased risk of severe or life-threatening treatment-related toxicity and tumor radioresistance pose challenges to re-irradiation. Because locoregional progression is the most common cause of death in patients with $\mathrm{HNC}$, obtaining local control may effect the survival of patients with locoregionally failed disease. In addition, local tumor progression may affect morbidity due to disfigurement in appearance, uncontrollable pain, cancer bleeding, infection, and impairment of speech and swallowing, thus resulting in a poor quality of life. Patients with recurrent or second primary HNC having a history of irradiation comprise a challenging heterogeneous group. Published data include diverse recurrent or second primary tumors in the extent and location, prior radiotherapy (RT) parameters, elapsed time since prior treatment, and extent and severity of normal tissue sequelae. Data in current literature on acute and late normal tissue recovery from prior treatment are not available. Lack of data pertaining to re-irradiation tolerance poses significant challenges or even 
leads to fear pertaining to meeting these patients daily in the clinic. Recently, high precision RT, including intensity-modulated radiotherapy (IMRT), has demonstrated the ability to reduce toxicity and improve disease control. Novel systemic agents and radiotherapy techniques, including stereotactic body RT (SBRT) and proton therapy, are also being actively explored.[2]

Salvage surgery is the standard for patients suitable for surgery, but it can be successfully performed only in $25 \%$ of patients. In a Phase II multicenter randomized trial (Radiation Therapy Oncology Group [RTOG] 9901 ), the outcome of chemotherapy treatment with postsurgical re-irradiation in recurrent disease was evaluated. [3] Overall, 130 patients who underwent salvage surgery were randomized to the observation and chemoradiotherapy arms. Local control and disease-free survival were increased in the chemoradiotherapy arm. [4,5]

Chang et al showed that age, Charlson comorbidity index score, clinical stage at first diagnosis, and recurrence-free interval were significant independent prognostic factors for overall survival (OS) of patients with recurrent head-and-neck squamous cell carcinoma (HNSCC). Regardless of the recurrence stage or site, salvage surgery is the recommended procedure. Re$\mathrm{RT}$ alone and concurrent chemoradiotherapy are more suitable for inoperable recurrent HNSCCs.[6]

The use of SBRT with re-irradiation in recurrent disease has demonstrated the advantages of better protection of organs at risk and higher doses in the target volume. The duration of treatment is shortened in patients with poor prognosis due to hypofractionation.

Unger et al reported the outcome of 65 patients treated with median 30 Gy (21-35) SBRT in 2-5 fractions (33 patients received concomitant chemotherapy). Median OS for all patients was 12 months, and 2-year OS and locoregional control (LRC) rates were $41 \%$ and $30 \%$, respectively. Complete response, partial response, and progressive disease rates were $54 \%, 27 \%$ and $20 \%$, respectively. Higher total dose, surgical resection, and nasopharynx site were significantly associated with improved LRC; surgical resection and non-squamous histology were associated with improved OS.[7] In a retrospective analysis of 46 patients in whom Cengiz et al performed a median 30 Gy (18-35 Gy) SBRT in 1-5 fractions, the OS at 1 year was $46 \%$. Complete response, partial response, and stable disease rates were $27 \%, 30 \%$, and $27 \%$, respectively.[8] Treatment responses were similar to those observed in other studies, but the late grade 4 toxicity rate was high. Of the 8 patients with late carotid blowout, 7 died due to carotid hemorrhage.[9]
The 1-year OS rate was $47 \%$ for a Phase II multicenter study in which Lartigau et al used concomitant cetuximab with 36 Gy SBRT in 6 fractions between 11 and 12 days for the treatment of patients with recurrent or newly diagnosed HNC. Complete response, partial response, and stable disease rates were $49 \%, 20 \%$, and $23 \%$, respectively. Grade 3/4 toxicity was seen in 32\% of patients. Only one patient died because of the choice of patients with the carotid artery wrapped around a fewer than third of the carotid artery, resulting in a carotid blowout.[10] Seventy patients in the Pittsburgh series were retrospectively matched and analyzed. Of these, 35 patients received concurrent cetuximab treatment with SBRT and the remaining patients received only SBRT. In the cetuximab group, OS was higher (24.5 vs. 14.8 months). Grade 3/4 toxicity was not different between the two groups.[11] Patient selection criteria, differences in tumor histology, radiotherapy fractionation, and dose differences make it difficult to compare studies. In conclusion, phase III multicenter trials are needed to demonstrate whether the 2-year median survival, particularly Heron et al's study, is reproducible.[12]

A multi-institutional study validated the recursive partitioning analysis (RPA) classification for patients with unresected recurrences treated with SBRT and compared outcomes of patients with unresected disease treated with IMRT.[13] Authors have analyzed 412 patients from 7 institutions in North America. RPA identified 3 prognostic subgroups: class I included patients $>2$ years from initial RT with resected tumors; class II included patients $>2$ years from initial RT with unresected tumors or those $\leq 2$ years without feeding tube or tracheostomy dependence; and the remaining patients formed class III. The authors highlighted the differences between the cohorts, a reflection of the selection bias inherent in retrospective studies where treatment is typically selected based on the baseline characteristics. SBRT-treated patients had a shorter interval between RT courses (1.2 years vs. 3.1 years) and were more likely to have prior chemotherapy ( $64 \%$ vs. 46\%) than IMRT-treated patients. After adjustment analysis, differences in OS and locoregional failure (LRF) between IMRT and SBRT were no longer statistically significant. In a second attempt to minimize bias introduced by baseline differences, a subgroup analysis by RPA class was performed. In the poorprognosis class III group, the investigators observed a statistically insignificant difference in 2-year OS with IMRT than with SBRT (16.2\% vs. 3.6\%). Among class II patients, IMRT was associated with a statisti- 
cally significantly better 2 -year OS rate compared with SBRT $(39.1 \%$ vs. $18.6 \%, \mathrm{P}<.001)$. This difference was attenuated substantially when further stratified by tumor size and SBRT dose. Specifically, patients with small tumors ( $\leq 25 \mathrm{~cm} 3$ or rT0-2) treated with $\geq 35$ Gy of SBRT had a 2-year OS rate closer to that of patients treated with IMRT (38.5\% vs. $50 \%)$. For patients with larger tumors treated with SBRT, the OS difference remained significantly worse than that in those treated with IMRT irrespective of the SBRT dose ( $28.2 \%$ with IMRT vs. $9.1 \%$ with SBRT $<35$ Gy or $8.8 \%$ with SBRT $\geq 35$ Gy). The rate of LRF mirrored that of OS, which is no surprise because LRF is frequently the ultimate cause of death for recurrent HNC. In terms of toxicity trade-off, the rate of acute grade 4 or 5 toxicity was low, but such toxicity was more common with IMRT ( $5.1 \%$ vs $0.5 \%$ ), and rates of grade $>3$ toxicity beyond 90 days were comparable after adjustment for competing risks of recurrence or death (12.4\% with IMRT vs. $11.6 \%$ with SBRT). Although the toxicity of IMRT and SBRT re-irradiation appeared similar, the study demonstrated differences in outcome between IMRT and SBRT, particularly among class II patients with larger tumors, which need to be evaluated in a prospective setting. Patterns-of-failure analysis after SBRT would be helpful to determine whether failures are marginal to the treated gross tumor volume, particularly among larger tumors that may require a larger clinical target volume margin. Besides the standard clinical examination and imaging, other methods are needed to help clinicians decide when a tumor can be treated with a minimal margin and when a larger margin is needed to account for subclinical spread. In addition, dose and fractionation may play a role in the observed OS and LRF differences between SBRT and IMRT.[14]

Carotid blowout syndrome and spinal cord myelopathy are rare and are late lethal side effects of re-irradiation.[15] For re-irradiation of the full cord cross-section at 2 Gy per day after prior conventionally fractionated treatment, cord tolerance appears to increase at least by $25 \% 6$ months after the initial course of RT based on animal and human studies. For partial cord irradiation as part of spine radiosurgery, a maximum cord dose of $13 \mathrm{~Gy}$ in a single fraction or 20 Gy in 3 fractions appears associated with a $<1 \%$ risk of injury.[16] Carotid blowout syndrome was observed in patients who were treated more than once in the study by Yazici et al. and for whom the maximum carotid artery dose was below 34 Gy.[17] However, Gebhardt et al. reported no carotid blowout syndrome below 47.6 Gy maximum point dose to carotid artery.[18]

\section{Conclusion}

In conclusion, stereotactic body radiotherapy is a promising therapy that is still developing in the treatment of HNCs that cannot be resected locally after definitive radiotherapy. In general, the 1-year survival rates following treatment are similar in conventional radiotherapy groups only with chemotherapy and chemotherapy. Because of the small volume of treatment in SBRT, it is difficult to compare SBRT with other palliative treatment methods in terms of toxicity. SBRT is more advantageous than other palliative treatment methods with a shorter treatment duration.

Peer-review: Externally peer-reviewed.

Conflict of Interest: None declared.

\section{References}

1. McDonald MW, Lawson J, Garg MK, Quon H, Ridge JA, Saba N, et al. ACR appropriateness criteria retreatment of recurrent head and neck cancer after prior definitive radiation expert panel on radiation oncology-head and neck cancer. Int J Radiat Oncol Biol Phys 2011;80(5):1292-8. [CrossRef]

2. Kim YS. Reirradiation of head and neck cancer in the era of intensity-modulated radiotherapy: patient selection, practical aspects, and current evidence. Radiat Oncol J 2017;35(1):1-15. [CrossRef]

3. Janot F, de Raucourt D, Benhamou E, Ferron C, Dolivet G, Bensadoun RJ, et al. Randomized trial of postoperative reirradiation combined with chemotherapy after salvage surgery compared with salvage surgery alone in head and neck carcinoma. J Clin Oncol 2008;26(34):5518-23. [CrossRef]

4. Popovtzer A, Gluck I, Chepeha DB, Teknos TN, Moyer JS, Prince ME, et al. The pattern of failure after reirradiation of recurrent squamous cell head and neck cancer: implications for defining the targets. Int J Radiat Oncol Biol Phys 2009;74(5):1342-7. [CrossRef]

5. Chen AM, Farwell DG, Luu Q, Cheng S, Donald PJ, Purdy JA. Prospective trial of high-dose reirradiation using daily image guidance with intensity-modulated radiotherapy for recurrent and second primary head-and-neck cancer. Int J Radiat Oncol Biol Phys 2011;80(3):669-76. [CrossRef]

6. Chang JH, Wu CC, Yuan KS, Wu ATH, Wu SY. Locoregionally recurrent head and neck squamous cell carcinoma: incidence, survival, prognostic factors, and treatment outcomes. Oncotarget 2017;8(33):55600-12.

7. Unger KR, Lominska CE, Deeken JF, Davidson BJ, 
Newkirk KA, Gagnon GJ, et al. Fractionated stereotactic radiosurgery for reirradiation of head-and-neck cancer. Int J Radiat Oncol Biol Phys 2010;77(5):14119. [CrossRef]

8. Cengiz M, Özyiğit G, Yazici G, Doğan A, Yildiz F, Zorlu F, et al. Salvage reirradiaton with stereotactic body radiotherapy for locally recurrent head-and-neck tumors. Int J Radiat Oncol Biol Phys 2011;81(1):104-9.

9. Vargo JA, Heron DE, Ferris RL, Rwigema JC, Kalash R, Wegner RE, et al. Examining tumor control and toxicity after stereotactic body radiotherapy in locally recurrent previously irradiated head and neck cancers: implications of treatment duration and tumor volume. Head Neck 2014;36(9):1349-55. [CrossRef]

10. Lartigau EF, Tresch E, Thariat J, Graff P, Coche-Dequeant B, Benezery $\mathrm{K}$, et al. Multi institutional phase II study of concomitant stereotactic reirradiation and cetuximab for recurrent head and neck cancer. Radiother Oncol 2013;109(2):281-5. [CrossRef]

11. Vargo JA, Ferris RL, Ohr J, Clump DA, Davis KS, Duvvuri $U$, et al. A prospective phase 2 trial of reirradiation with stereotactic body radiation therapy plus cetuximab in patients with previously irradiated recurrent squamous cell carcinoma of the head and neck. Int J Radiat Oncol Biol Phys 2015;91(3):480-8. [CrossRef]

12. Heron DE, Rwigema JC, Gibson MK, Burton SA, Quinn AE, Ferris RL. Concurrent cetuximab with stereotactic body radiotherapy for recurrent squamous cell carcinoma of the head and neck: a single institution matched case-control study. Am J Clin Oncol 2011;34(2):165-72.

13. Vargo JA, Ward MC, Caudell JJ, Riaz N, Dunlap NE, Isrow D, et al. A Multi-institutional Comparison of SBRT and IMRT for Definitive Reirradiation of Recurrent or Second Primary Head and Neck Cancer. Int J Radiat Oncol Biol Phys 2018;100(3):595-605. [CrossRef]

14. Margalit DN, Wong SJ. Reirradiation for Head and Neck Cancer: The Who and the How. Int J Radiat Oncol Biol Phys 2018;100(3):618-20. [CrossRef]

15. Powitzky R, Vasan N, Krempl G, Medina J. Carotid blowout in patients with head and neck cancer. Ann Otol Rhinol Laryngol 2010;119(7):476-84. [CrossRef]

16. Kirkpatrick JP, van der Kogel AJ, Schultheiss TE. Radiation dose-volume effects in the spinal cord. Int J Radiat Oncol Biol Phys 2010;76(3 Suppl):S42-9. [CrossRef]

17. Yazici G, Sanlı TY, Cengiz M, Yuce D, Gultekin M, Hurmuz P, et al. A simple strategy to decrease fatal carotid blowout syndrome after stereotactic body reirradiaton for recurrent head and neck cancers. Radiat Oncol 2013;8:242. [CrossRef]

18. Gebhardt BJ, Vargo JA, Ling D, Jones B, Mohney M, Clump DA, et al. Carotid Dosimetry and the Risk of Carotid Blowout Syndrome After Reirradiation With Head and Neck Stereotactic Body Radiation Therapy. Int J Radiat Oncol Biol Phys 2017 Dec 5 [Epub ahead of print], doi: 10.1016/j.ijrobp.2017.11.045. [CrossRef] 